\title{
A geometric approach to the modelling of critical phenomena for a spray combustion model
}

\author{
Elena Shchepakina ${ }^{1, *}$ \\ ${ }^{1}$ Samara National Research University, 34, Moskovskoye shosse, Samara 443086, Russia
}

\begin{abstract}
The paper is devoted to the modelling of the critical phenomena in multiscale combustion models. Such models are usually described by singularly perturbed systems of differential equations to reflect the significant distinction in characteristic relaxation times of different physicochemical processes. The paper proposes an approach for modelling of critical phenomena on the basis of the geometric asymptotic method of invariant manifolds. The critical phenomenon means as a sharp change in the dynamics of the process under consideration. As an illustration of this approach a dynamic model of fuel spray ignition and combustion is considered. The realizability conditions for the critical regime is obtained in the form of the asymptotic expression for the control parameter. The main feature of the critical regime is that during it the temperature of the combustible mixture can reach a high value within the framework of a safe process. It is shown that the critical regime plays the role of a watershed between the slow combustion regimes and the thermal explosion.
\end{abstract}

\section{Introduction}

A wide spectrum of thermophysical and chemical processes occurring during combustion, especially in multiphase media, is characterized by a significant difference in time scales (for example, temperature changes vs. concentration changes). Therefore, the singularly perturbed systems of differential equations are used to model combustion processes. For studying such systems different asymptotic methods are usually applied. The asymptotic geometric method used in this paper is based on the theory of invariant manifolds (see, for instance, [1] and the references therein). This method allows us to replaces the original system by another system on an invariant manifold of lower dimension. The lowering of the dimension occurs due to the decomposition of the original system in the vicinity of the integral surface into the independent "slow" subsystem and the "fast" subsystem. If the slow invariant manifold is attractive, then the analysis of the original system can be replaced by the analysis of the slow subsystem retaining a desired degree of accuracy. The use of such approach makes it possible not only to substantially simplify the initial system by reducing its dimension and by eliminating the computational rigidity, but it also gives us a full picture of the dynamics of the physical processes and provides an effective tool for

\footnotetext{
* Corresponding author: shchepakina@yahoo.com
} 
modelling the critical phenomena in these processes. The critical regime corresponds to a chemical reaction separating the domains of self-accelerating reactions and domains of safe reactions. This regime is modelled by a unique trajectory that contains an unstable invariant manifold.

\section{Spray ignition model}

The model of the ignition and combustion of a fuel spray is suggested using an adiabatic approach [2-5]. The fuel spray is considered as a two-phase medium: combustible gas mixture - combustible liquid droplets. The pressure change in the reaction volume and its effect on the combustion process is assumed a slight and is omitted. The usual assumption is made that the thermal conductivity of the liquid phase is much greater than that in the gas phase. Thus, the heat transfer coefficient in the liquid gas mixture is supposed to be defined by the thermal properties of the gas phase. The drop boundary is assumed to be on a saturation line, i.e., the liquid temperature is constant and is equal to the liquid saturation temperature. The combustion reaction is modelled as a first order, highly exothermic chemical reaction. The model is built with the usual assumptions of the theory of combustion processes in chemical homogeneity at each point of the reaction vessel and in the dimensionless form has the form [4]:

$$
\begin{gathered}
\gamma \frac{d \theta}{d \tau}=\eta \exp \left(\frac{\theta}{1+\beta \theta}\right)-\varepsilon_{1} r \theta(1+\beta \theta), \\
\frac{d r^{3}}{d \tau}=-\varepsilon_{1} \varepsilon_{2} r \theta, \\
\frac{d \eta}{d \tau}=-\eta \frac{1}{1+\beta \theta} \exp \left(\frac{\theta}{1+\beta \theta}\right)+\varepsilon_{1} \psi r \theta,
\end{gathered}
$$

where $\theta$ is the dimensionless fuel gas temperature; $r$ is the dimensionless radius of the drops; $\eta$ is the dimensionless concentration of flammable gas; $\tau$ is the dimensionless time; $\gamma$ is the dimensionless parameter equal to the final dimensionless adiabatic temperature thermally isolated system after the explosion; $\beta$ gives the initial temperature; $\varepsilon_{1}, \varepsilon_{2}$ characterize the interaction between the gas and liquid phases; $\psi$ is a parameter characterizing the ratio of the energy of combustion gas mixture to the liquid evaporation energy.

The initial conditions for the equations (1)-(3) are:

$$
\theta(0)=0, r(0)=1, \eta(0)=1 \text {. }
$$

Appropriate combination of equations (1)-(3) and integration over time yields the following energy integral

$$
\eta-1+\frac{\gamma}{\beta} \ln (1+\beta \theta)+\frac{\psi-1}{\varepsilon_{2}}\left(r^{3}-1\right)=0,
$$

which allows to reduce the order of the system (1)-(3) to the form

$$
\begin{gathered}
\gamma \frac{\mathrm{d} \theta}{\mathrm{d} \tau}=\left[1-\frac{\gamma}{\beta} \ln (1+\beta \theta)-\frac{\psi-1}{\varepsilon_{2}}\left(r^{3}-1\right)\right] \exp \left(\frac{\theta}{1+\beta \theta}\right)-\varepsilon_{1} \mathrm{r} \theta(1+\beta \theta) \\
\frac{\mathrm{dr}^{3}}{\mathrm{~d} \tau}=-\varepsilon_{1} \varepsilon_{2} \mathrm{r} \theta .
\end{gathered}
$$


Thus, the dynamic of the system depends on five dimensionless parameters: $\beta \ll$ $1, \gamma \ll 1, \varepsilon_{1}, \varepsilon_{2}, \psi$. The degenerate equation, which follows from the fast subsystem (4) for $\gamma=0$, describes a slow curve

$$
\Lambda(\theta, r)=\left[1-\frac{\gamma}{\beta} \ln (1+\beta \theta)+\frac{(\psi-1)\left(1-r^{3}\right)}{\varepsilon_{2}}\right] \exp \left(\frac{\theta}{1+\beta \theta}\right)-\varepsilon_{1} r \theta(1+\beta \theta)=0
$$

in the phase plane. The flow of the system (4), (5) near the slow curve has a velocity of order of one as $\gamma \rightarrow 0$ while far from the slow curve the variable $\theta$ is changed very fast. In $\gamma$ - neighborhood of the slow curve there exists a slow invariant manifold of the system which is defined as an invariant surface of slow motions.

The set of points on the slow curve at which $\partial \Lambda / \partial \theta<0(\partial \Lambda / \partial \theta>0)$ forms the stable (unstable) part of the slow curve. The stable and unstable parts of the slow curve are the zero-order approximations $(\gamma=0)$ of the stable (or attractive) and unstable (or repulsive) slow invariant manifolds of the system (4), (5), respectively.

For $0<\psi<1-\varepsilon_{2}$ the slow curve has the shape similar to that shown in Fig. 1. The part $P T$ of the slow curve is stable while the part $T Q$ is unstable. The ordinate of the point $T$ depending on the parameters values can be equal to 1 or be greater (or less) than 1 . If the point $T$ has an ordinate greater than 1 then a trajectory of the system starting from the initial point tends to stable part $P T$ of the slow curve and follows it to the origin. This is the case of the slow combustion regime; see the trajectory CTP in Figure 1.

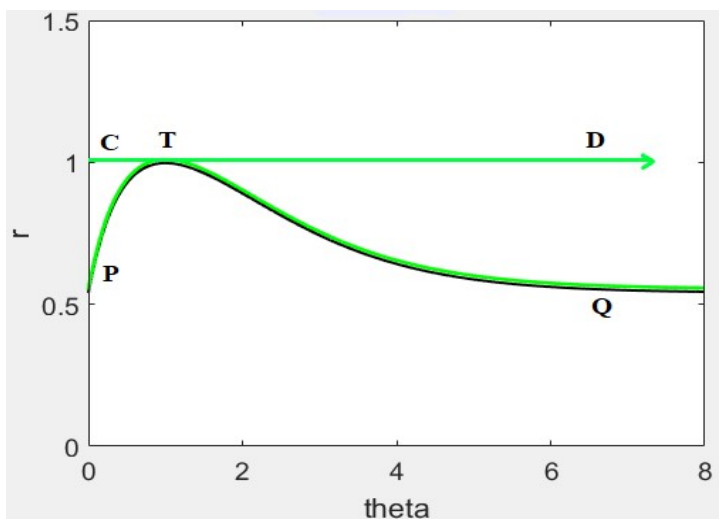

Fig. 1. Slow curve (black) and the trajectories (green) of (4), (5) in limit case $(\gamma=0)$

If the point $T$ has an ordinate less than 1 then a trajectory of the system starting from the initial point will pass beyond the basin of attraction of the stable part of the slow curve. This is the case of the explosion regime; see the trajectory $C D$ in Figure 1.

The critical regime corresponds to the case when the trajectory of the system falls into a small vicinity of the point $T$ and passes along the unstable part $T Q$ of the slow curve; see the trajectory $C T Q$ in Figure 1. The critical regime separates the explosive regimes from the safe combustion modes $[1,6]$. The crucial result is that the unstable slow manifold may be used to construct the critical trajectory $C T Q$ and to calculate the corresponding value of a control parameter, say, $\varepsilon_{1}$, of the system. For this goal we use the asymptotics proposed in [7].

The part $C T$ of the critical trajectory can be represented in the form:

$$
r(\theta, \gamma)=r^{*}+\gamma^{\frac{2}{3}} \Gamma_{0}^{\frac{2}{3}} \Omega \operatorname{sign} f\left(r^{*}, \theta^{*}\right)+\frac{1}{3} \gamma \ln \frac{1}{\gamma} \Gamma_{1} \operatorname{sign} f\left(r^{*}, \theta^{*}\right)+O(\gamma),
$$

where $r^{*}, \theta^{*}$ denote the coordinates of the point $T$, 


$$
\begin{gathered}
g(\theta, r)=\left[-1+\frac{\gamma}{\beta} \ln (1+\beta \theta)+\frac{\psi-1}{\varepsilon_{2}}\left(r^{3}-1\right)\right] \exp \left(\frac{\theta}{1+\beta \theta}\right)+\varepsilon_{1} r \theta(1+\beta \theta), \\
f(\theta, r)=\varepsilon_{1} \varepsilon_{2} r \theta, \quad \omega=2.338107, \quad \Gamma_{0}=\sqrt{\frac{2}{\left|g_{\theta \theta}\left(r^{*}, \theta^{*}\right) g_{r}\left(r^{*}, \theta^{*}\right)\right|}}\left|f\left(r^{*}, \theta^{*}\right)\right|, \\
\Gamma_{1}=\frac{6 g_{\theta \theta}\left(r^{*}, \theta^{*}\right) f_{\theta}\left(r^{*}, \theta^{*}\right)-2 g_{\theta \theta \theta}\left(r^{*}, \theta^{*}\right) f\left(r^{*}, \theta^{*}\right)}{3 g_{\theta \theta}^{2}\left(r^{*}, \theta^{*}\right)} .
\end{gathered}
$$

The functions $f$ and $g$ in the representation (6) are the right parts of the system (4), (5) after the transition to the inverse time that make the part $T Q$ of the slow curve stable. The coordinates $r^{*}, \theta^{*}$ can be calculated from the system $g\left(r^{*}, \theta^{*}\right)=g_{\theta}\left(r^{*}, \theta^{*}\right)=0$.

Substituting all the found values into (6) and setting $r=1$ since the point $C$ has the coordinate $r=1$, we obtain the equation for calculation of the critical values of the parameter $\varepsilon_{1}=\varepsilon_{1}^{*}$ in the form of asymptotic representation

$$
\varepsilon_{1}^{*}=\varepsilon_{10}+\gamma^{\frac{2}{3}} \varepsilon_{11}+\gamma \ln \frac{1}{\gamma} \varepsilon_{12}+O(\gamma) .
$$

The direct calculation gives

$$
\begin{gathered}
\varepsilon_{10}=\frac{p e(\psi-1)}{\varepsilon_{2}}, \varepsilon_{11}=-54 \Omega(\psi-1)^{3^{3}} \sqrt{\frac{2}{9}} \mu_{1}^{\frac{4}{3}} \mu_{23}^{-\frac{2}{3}} \varepsilon_{10}^{-\frac{4}{3}} \varepsilon_{2}^{-\frac{4}{3}} \\
\times\left\{(1-6 \beta)\left[\frac{\psi-1}{\varepsilon_{2}}\left(\mu_{23}^{3}-1\right)-1\right] \mathrm{e}+2 \varepsilon_{10} \mu_{23} \beta\right\}^{-\frac{1}{3}}\left[3 \mu_{23}^{2} \mathrm{e} \frac{\psi-1}{\varepsilon_{2}}+\varepsilon_{10}(1+\beta)\right]^{-\frac{1}{3}} \\
\varepsilon_{12}=\frac{\varepsilon_{10} \varepsilon_{2}}{3 \mu_{23}}\left\{(1-14 \beta)\left[\frac{\psi-1}{\varepsilon_{2}}\left(\mu_{23}^{3}-1\right)-1\right] \mathrm{e}+4 \varepsilon_{10} \mu_{23} \beta\right\} \\
\times\left\{(1-6 \beta)\left[\frac{\psi-1}{\varepsilon_{2}}\left(\mu_{23}^{3}-1\right)-1\right] \mathrm{e}+2 \varepsilon_{10} \mu_{23} \beta\right\}^{-2},
\end{gathered}
$$

where

$$
q=\frac{\varepsilon_{2}+\psi-1}{\psi-1}, \mu_{1}=\sqrt{\frac{q^{2}}{4}+\frac{\varepsilon_{10}^{3} \varepsilon_{2}^{3}}{27(\psi-1)^{3}}}, \mu_{2}=\sqrt[3]{\frac{q}{2}+\mu_{1}}, \mu_{3}=\sqrt[3]{\frac{q}{2}-\mu_{1}}, \mu_{23}=\mu_{2}+\mu_{3},
$$

and $p$ is a root of the equation

$$
1=\sqrt[3]{\frac{q}{2}+\sqrt{\frac{q^{2}}{4}+\frac{p^{3}}{27}}}+\sqrt[3]{\frac{q}{2}-\sqrt{\frac{q^{2}}{4}+\frac{p^{3}}{27}}}
$$

The value $\varepsilon_{1}=\varepsilon_{1}^{*}$, at which the trajectory of (4), (5) contains the unstable manifold is said to be critical, i.e. this regime is not a slow combustion regime, since the temperature achieves a high value, and is not explosive, as the temperature increases at the tempo of the slow variable as this is the slow manifold. The value of $\varepsilon_{1}=\varepsilon_{1}^{*}$, gives the critical trajectory which separates the explosion modes $\left(\varepsilon_{1}<\varepsilon_{1}^{*}\right)$ from the slow regimes $\left(\varepsilon_{1}>\varepsilon_{1}^{*}\right)$ which are characterized by a slowdown of the reaction with small degrees of conversion and heating up is limited from above by $\theta<\theta^{*}$ at $T$. 
Figure 2 shows the trajectory of the system (4), (5) and the solutions of the system (1)(3) in the case of critical regime for $\varepsilon_{1}=2.2100108, \gamma=0.01, \varepsilon_{1}=3.5, \varepsilon_{2}=0.8, \beta=$ $0.05, \psi=0.19$.
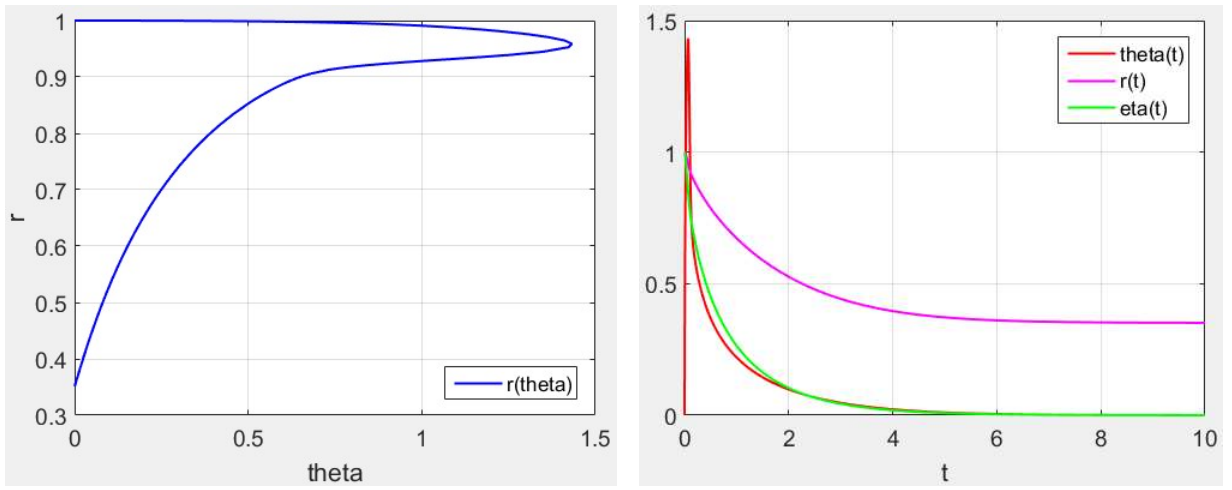

Fig. 2. Trajectory of (4), (5) and the solutions of (1)-(3) in the case of critical regime.

\section{Conclusions}

The dynamic model of a fuel spray ignition was studied by the methods of the geometric theory of singular perturbations. The realizability conditions for the critical regime was obtained in the form of the asymptotic expression for the control parameter. The main feature of the critical regime is that during it the temperature of the combustible mixture can reach a high value within the framework of a safe process. It was shown that the critical regime plays the role of a watershed between the slow combustion regimes and the thermal explosion.

The analytical calculations presented in this paper are of utmost importance for the realization of safe high-temperature processes.

This work was supported by the Ministry of Education and Science of the Russian Federation as part of a Program "Research and Development in Priority Areas of Advancement of the Russian Scientific and Technological Complex for 2014-2020" (RFMEFI58716X0033).

\section{References}

1. E. Shchepakina, V. Sobolev, M.P. Mortell, Singular Perturbations. Introduction to System Order Reduction Methods with Applications (Springer, Cham, 2014)

2. R. Stone, Introduction to Internal Combustion Engines (The MacMillan Press Ltd, 2nd Ed., 1992)

3. S. Sazhin, Droplets and Sprays (Springer, London, 2014)

4. I. Goldfarb, V. Gol'dshtein, I. Shreiber, A. Zinoviev, Proceedings of the 26th Symposium on Combustion, 1557 (The Combustion Institute, Pittsburgh, 1996)

5. I. Goldfarb, V. Gol'dshtein, G. Kuzmenko, S. Sazhin, Combust. Theory Modell. 3, 769 (1999)

6. V.A. Sobolev, E.A. Shchepakina, Combust. Explos. Shock Waves 29(3) 378 (1993)

7. E.F. Mishchenko, N. Kh. Rozov, Differential Equations with Small Parameters and Relaxation Oscillations (Plenum Press, New York, 1980) 